\title{
The Use of Interval-Training
}

Methods by Coaches of

Well-Trained Middle- to

Long-Distance Runners

\author{
Arran Parmar ${ }^{1}$, Thomas W. Jones ${ }^{1}$, Philip, R. Hayes ${ }^{1}$ \\ ${ }^{1}$ Department of Sport, Exercise and Rehabilitation, Faculty of Health and Life Sciences, Northumbria University, \\ Northumberland Building, Newcastle-upon-Tyne, UK
}

\section{ABSTRACT}

Limited research exists on how coaches of welltrained middle- to long-distance runners implement interval-training (IT) methods. IT interventions within research focus on physiological measures whereas coaches focus on performance, leading to a disconnect between the IT methods utilized in research and practice. This study aimed to identify how coaches of well-trained middle- to long-distance runners implement IT methods within the training regimen. A survey was developed that comprised 5 sections: participant demographics, the use of IT, the type and characteristics of IT, recovery from IT, and reasons for including IT. Thirty (29 male, 1 female) coaches completed the survey. All coaches prescribed short, medium and long work intervals into the training regimen using race paces of $800 \mathrm{~m}, 3000 \mathrm{~m}$, and $10000 \mathrm{~m}$, respectively, to prescribe intensity. Active recovery modalities were prescribed by the majority of coaches between sets and repetitions of all types of work intervals. The majority of coaches periodized the type of work interval prescribed relative to the competition date, with 1-2 IT sessions prescribed per week, year-round. Most coaches reported learning about IT primarily from their own training and coaching books, however, the most valuable sources of education for IT were ranked as scientific literature, coaching courses and workshops.

Keywords: Endurance, Performance, Running, Physiology, Intermittent

\section{INTRODUCTION}

Success in middle- to long-distance running is known to be determined by physiological parameters such as maximal aerobic power (VO2max), sustainable percentage of $\mathrm{VO}_{2 \max }\left(\% \mathrm{VO}_{2 \max }\right)$, velocity at lactate threshold $(\mathrm{VLT})$, velocity at $\mathrm{VO}_{2 \max }\left(\mathrm{VVO}_{2 \max }\right)$, and running economy $(R E)^{1}$. Training methods to improve these determinants of performance have been developed with varying success, with two modes of training typically identified: continuous training (CT) and interval-training (IT)2. Both methods of training elicit physiological adaptations facilitating endurance performance, however, the physiological structures targeted differ ${ }^{1,2}$. Continuous training is characterized by long duration sub-maximal efforts (below the second ventilatory threshold $\left(\mathrm{VT}_{2}\right)$ ), whereas IT is characterized by repeated high-intensity efforts (above $\mathrm{VT}_{2}$ ) interspersed with periods of recovery ${ }^{3,4}$. Although much is known about the physiological effects of training on endurance performance, a lack of information exists relating to the interval-training practices of elite endurance runners.

The use of IT was popularized in the 1950's by both coaches and athletes reportedly implementing high volumes of short work intervals (SWI) (e.g. $20 \mathrm{x}$ $400 \mathrm{~m}$ with $200 \mathrm{~m}$ recovery jogs) almost exclusively 5 . Conversely, the 1960's saw coaches implement high proportions of CT in relation to IT, with the prescribed IT methods periodized by implementing low volumes of high-intensity, long work intervals 
(LWI) before progressing to shorter, intense sprints of $10-15 s^{3,6}$. From the 1980's to the present day, the periodized prescription of IT methods was advanced further by varying training intensity on a daily basis; with reported training regimes of international level runners including IT methods 3-5 times a week, in addition to high volumes of lower intensity, longer duration CT sessions ${ }^{3}$. The IT performed was comprised of various combinations of intensities, repetitions, durations and volumes, with the IT interventions prescribed reportedly based on race performances in well-trained middle- to longdistance runners ${ }^{7}$. This variety in the design of the IT interventions prescribed indicates a consensus of the most effective IT methods has not been reached despite their popularity ${ }^{3}$. The lack of agreement in the design of IT interventions also appears evident in the periodization of IT methods within the overall training regime. Research documenting the training intensity distribution (TID) of elite endurance runners have commonly reported the use of both a polarized TID (approximately $80 \%$ below the first ventilatory threshold $\left[\mathrm{VT}_{1}\right.$ ] and $20 \%$ above $\mathrm{VT}_{2}$ ) and a threshold TID (high volumes of training performed between VT. and $\left.\mathrm{VT}_{2}\right)^{8}$. Scientific literature suggests a polarized TID model to be effective for improving key physiological determinants of endurance performance, however, a high proportion of elite endurance runners adopt a threshold TID despite this approach considered more demanding yet less effective for improving endurance performance ${ }^{4,8}$. Furthermore, the documented training practices of elite endurance runners have been limited to small samples 9-13, short time periods ${ }^{10,11,13}$, generalizations of training distances $^{9,12}$, and generalizations of training methods ${ }^{10-14}$. The conclusions drawn from these reports are speculative at best, leaving much unknown about the implementation of IT methods into the training regime of well-trained runners.

The efficacy of IT to improve parameters of middleto long-distance running performance has been explored within research, primarily focused on improving $\mathrm{VO}_{2 \max }{ }^{15-17}$. Research suggests IT to be superior to CT methods for improving $\mathrm{VO}_{2 \max }$ irrespective of the type of intervals implemented, however, these conclusions have been reported in recreationally- to moderately-trained populations ${ }^{15,17}$. The few studies conducted on well-trained runners performing IT interventions to improve $\mathrm{VO}_{2 \max }$ show inconclusive results ${ }^{1,18}$. The efficacy of IT remains speculative when applied to well-trained middle- to long-distance runners; not only due to the traininglevel of the athletes included in the studies, but the design of the IT interventions. IT methods are able to increase the total time spent at an elevated intensity compared to CT methods, thus eliciting greater adaptations due to the ability to maximally stress the cardiopulmonary system ${ }^{2,16}$. This has led to IT interventions within research primarily focusing on the physiological responses to IT, with specific priority placed upon maximizing the time spent near the intensity of $\mathrm{VO}_{2 \max }{ }^{1}$

The physiological emphasis placed upon the IT methods used within scientific literature are at a discourse with the more performance orientated approach used by coaches in practice, both as it relates to the periodization of training intensity and the IT session structure. Moreover, information gathered on the IT methods implemented by coaches are generally incomplete, with IT methods typically reported during peaking phases of training and generally in small samples of elite athletes, meaning a broader and deeper view is required to fully understand how coaches implement IT. This disparity in the approach taken by researchers compared to coaches in practice, and the limited information on coaching practices, highlights a clear disconnect exists between the IT methods implemented within the training regime of welltrained runners in practice and research. Therefore, the aim of this study was to identify how and why coaches of well-trained middle- to long-distance runners implement IT methods.

\section{MATERIALS AND METHODS}

\section{Experimental approach to the problem}

A 5-part, 32-section survey was administered to middle- to long-distance running coaches $(800 \mathrm{~m}$ to ultra-distance) to anonymously identify how IT methods are implemented into the training regimen of the runners they coach. Coaches of runners, rather than runners themselves were chosen to avoid repetition, where several runners in one group would all complete the survey. The survey was designed in collaboration with a running coach, exercise physiologist and an academic with previous experience of conducting surveys. The survey was made available on-line via the "Online Survey platform" for a period of 9-months (February 2019 October 2019) to accrue responses internationally. It was hypothesized that this study would provide a comprehensive view of the implementation of IT methods in well-trained middle- to long-distance runners. 


\section{Participants}

Prior to the launch of the survey, the institutional research ethics committee granted ethical approval. The survey was distributed online via social networking websites, public running club/ organization websites, and emails sent to coaches, clubs and contacts worldwide acquired by the lead investigator. The title page of the survey included information on the purpose of the study and outlined the risks and benefits of participation. Following the title page, a statement of informed consent was required to be agreed upon before progressing to the survey questions. Throughout the data collection period, the use of online social networking sites to accrue responses resulted in a total of 35,002 impressions, 653 total engagements, 46 clicks on the survey URL, and 29 'likes'.

\section{Procedures}

The survey contained 5 sections with both fixedresponse and open-ended questions that took 20-30 minutes for respondents to complete. Section one of the survey identified participant demographics and section two contained questions relating to the use of IT methods within the training regimen. Section three of the survey contained questions relating to the type and characteristics of the IT methods used. The fourth section of the survey identified how recovery from IT sessions was prescribed and implemented, and the final section contained questions relating to the reasons why IT methods are included in the training regimen. Coaches were asked to provide a general indication of their IT practices in response to the survey questions irrespective of the competitive season or climate.

\section{Statistical Analyses}

The survey contained both fixed-response and open-ended questions. Fixed-response questions generated categorical and ordinal data. Answers to open-ended questions were analysed according to methods by Patton 19 by establishing higherorder themes via inductive content analysis using the qualitative analysis software "NVivo 12". Higherorder themes were individually established and then refined until agreement was reached at all levels of analysis. Agreed higher-order themes then underwent deductive analysis to confirm all raw data themes were represented.

\section{RESULTS}

\section{Participant demographics}

Thirty coaches provided responses to the survey (29 male, 1 female). The age range and coaching experience of the respondents are displayed in Table 1, and the coaching qualifications possessed are detailed in Table 2. Four of the coaches were based in the United States of America, 1 in Colombia, 1 in Chile, 1 in the Netherlands, 1 in Australia, and 22 in the United Kingdom. Of the 30 responses, 8 coaches identified as being independent and/ or freelance coaches with no affiliation to specific running clubs, and 22 coaches identified as being affiliated with local $(n=18)$ and national $(n=4)$ running clubs. Coaches were asked to detail their athlete's best performance times for the events they coached their athletes in, which are expressed as a percentage of the 2019 world record, and as the score achieved in the International Association of Athletics Federation (IAAF) scoring tables where applicable (Table 3A). Some respondents indicated coaching junior athletes (under 17), with these performance times reported in Table 3B. In addition to the events and performance times presented in Table 3A, coaches also reported the performance times of 7 uncategorized events. Specifically, a 5-mile run (1 Male, 26 min $40 \mathrm{~s}$ ), 6-mile river run (1 Male, $35 \mathrm{~min} 24 \mathrm{~s}$ ), $25 \mathrm{~km}$ trail run (1 Male, $3 \mathrm{~h} 2 \mathrm{~min}$ $22 \mathrm{~s}$ ), $70 \mathrm{~km}$ ultra (1 Male, $9 \mathrm{~h} 53 \mathrm{~min} 47 \mathrm{~s}$ ), $84 \mathrm{~km}$ ultra ( 1 Male, $9 \mathrm{~h} 10 \mathrm{~min} 48 \mathrm{~s}), 85 \mathrm{~km}$ ultra (1 Male, $15 \mathrm{~h} 38 \mathrm{~min} 9 \mathrm{~s}$ ), and a 120km ultra (1 Female, $26 \mathrm{~h}$ $39 \min 44 \mathrm{~s})$. 
Table 1. Age group, competitive level of athlete, and event distance currently coached by the respondents

\begin{tabular}{|c|c|c|c|c|c|c|}
\hline Age group & Under 30 & $31-40$ & $41-50$ & $51-60$ & $61-70$ & Over 70 \\
\hline$n$ & 2 & 7 & 4 & 7 & 8 & 2 \\
\hline $\begin{array}{c}\text { Competitive } \\
\text { Level }\end{array}$ & $\begin{array}{l}\text { Non-Compet- } \\
\text { itive }\end{array}$ & Club & County & Regional & National & International \\
\hline$n$ & 11 & 21 & 15 & 18 & 19 & 12 \\
\hline $\begin{array}{c}\text { Event Dis- } \\
\text { tance }\end{array}$ & $\begin{array}{c}\text { Middle } \\
\text { Distance } \\
(800 \mathrm{~m}-3000 \mathrm{~m})\end{array}$ & $\begin{array}{c}\text { Long Dis- } \\
\text { tance } \\
\text { (5000m-half } \\
\text { marathon) }\end{array}$ & Marathon & $\begin{array}{l}\text { Ultra-dis- } \\
\text { tance }\end{array}$ & Fell / Trail & \\
\hline$n$ & 16 & 23 & 15 & 3 & 2 & \\
\hline
\end{tabular}

Respondents detailed coaching more than one competitive level of athlete and event distance

Table 2. Coaching qualifications held by the respondents

\begin{tabular}{|c|c|c|}
\hline Qualification & $n$ & Selected raw data representing responses to this question \\
\hline EA Event Group Endurance & 7 & \\
\hline EA Leadership in Running Fitness & 5 & \\
\hline EA Coach in Running Fitness & 5 & \\
\hline EA Athletic Coach & 4 & \\
\hline IAAF Level 2 & 3 & \\
\hline USATF Level 1 & 3 & \\
\hline IAAF Level 4 & 2 & \\
\hline EA Coaching Assistant & 2 & \\
\hline USATF Level 2 & 2 & \\
\hline IAAF Level 1 & 1 & \\
\hline IAAF Level 5 & 1 & \\
\hline USATF Level 3 & 1 & \\
\hline Other & 5 & $\begin{array}{l}\text { - Physical culture, sports and recreation of Santo Tomás } \\
\text { - Sniversity Colombia; Running and trail running specialist } \\
\text { Spanish Swimming Federation Level } 3 \text { coach and Spanish } \\
\text { - } \quad \text { USTiathlon Federation Level } 3 \text { coach } \\
\text { - } \quad \text { Post-graduate diploma in elite sports coaching and UKA } \\
\text { Level } 3 \text { Performance Coach } \\
\text { - Coach from The Netherlands, coached National and Inter- } \\
\text { national level athletes competing at World Championships } \\
\text { and Olympic Games }\end{array}$ \\
\hline
\end{tabular}

Respondents detailed possessing more than one coaching qualification.

*IAAF = International association of athletics federation; $E A=$ England Athletics; USATF = United States of America Track and Field; USTFCCCA = United States Track and Field Cross Country Coaches Association; $U K A=$ United Kingdom Athletics. 
Table 3A. Best performance times in various events of the athlete's coaches currently support

Male

\begin{tabular}{|c|c|c|c|c|c|c|c|c|}
\hline \multirow[b]{2}{*}{ Event } & & \\
\hline & $n$ & $\begin{array}{l}\text { Performance } \\
\text { Time (min:s.ms } \\
\text { or h:min:s) }\end{array}$ & $\begin{array}{c}\text { Percentage of } \\
2019 \text { WR }\end{array}$ & IAAF points & $n$ & $\begin{array}{c}\text { Performance } \\
\text { Time (min:s.ms or } \\
\text { h:min:s) }\end{array}$ & $\begin{array}{c}\text { Percentage of } \\
2019 \text { WR }\end{array}$ & IAAF points \\
\hline $400 m$ & 0 & $\mathrm{n} / \mathrm{a}$ & $\mathrm{n} / \mathrm{a}$ & $\mathrm{n} / \mathrm{a}$ & 0 & $\mathrm{n} / \mathrm{a}$ & $\mathrm{n} / \mathrm{a}$ & $\mathrm{n} / \mathrm{a}$ \\
\hline $1500 \mathrm{~m}$ & 8 & $03: 50.8 \pm 0: 11.8$ & $91.9 \pm 4.7$ & $972 \pm 148$ & 2 & $04: 18.5 \pm 0: 33.2$ & $86.6 \pm 6.2$ & $989 \pm 139$ \\
\hline 1 mile & 0 & $\mathrm{n} / \mathrm{a}$ & $\mathrm{n} / \mathrm{a}$ & $\mathrm{n} / \mathrm{a}$ & 1 & 05:35.0† & 75.3† & 699† \\
\hline $3000 \mathrm{~m}$ & 1 & 09:15.0† & $81.5+$ & $661 \dagger$ & 2 & $09: 46.0 \pm 0: 12.7$ & $85.1 \pm 1.8$ & $957 \pm 39$ \\
\hline $5000 \mathrm{~m}$ & 10 & $17: 00.8 \pm 03: 06.0$ & $77.9 \pm 13.2$ & $574 \pm 388$ & 8 & $19: 09.1 \pm 03: 49.6$ & $77.3 \pm 13.7$ & $767 \pm 322$ \\
\hline $10000 \mathrm{~m}$ & 9 & $32: 34.8 \pm 04: 10.6$ & $76.2 \pm 18.8$ & $784 \pm 286$ & 6 & $35: 29.0 \pm 03: 31.0$ & $84.3 \pm 7.7$ & $969 \pm 163$ \\
\hline $15000 \mathrm{~m}$ & 1 & 45:29.0† & $91.9+$ & $989+$ & 1 & 50:19.0† & $88.1 \dagger$ & $1105 \dagger$ \\
\hline 10 miles & 0 & $\mathrm{n} / \mathrm{a}$ & $\mathrm{n} / \mathrm{a}$ & $\mathrm{n} / \mathrm{a}$ & 1 & 01:10:39† & $71.5+$ & $645 \dagger$ \\
\hline Half-MAR & 8 & $01: 29: 29 \pm 10: 24.3$ & $74.2 \pm 10.2$ & $551 \pm 295$ & 4 & $01: 16: 37 \pm 06: 03.4$ & $85.8 \pm 6.7$ & $995 \pm 135$ \\
\hline
\end{tabular}

Table 3B. Best performance times in various events of the junior athlete's (under 17) coaches currently support

\begin{tabular}{cccccc} 
& \multicolumn{2}{c}{ Junior Male } & & \multicolumn{2}{c}{ Junior Female } \\
\cline { 2 - 3 } \cline { 5 - 6 } Event & $\boldsymbol{n}$ & $\begin{array}{c}\text { Performance } \\
\text { Time (min:s.ms) }\end{array}$ & & $\boldsymbol{n}$ & $\begin{array}{c}\text { Performance } \\
\text { Time (min:s.ms) }\end{array}$ \\
\hline $400 \mathrm{~m}$ & 0 & $\mathrm{n} / \mathrm{a}$ & & 1 & $01: 00.1 \dagger$ \\
$800 \mathrm{~m}$ & 4 & $01: 54.2 \pm 0: 01.9$ & & 2 & $02: 18.0 \pm 0: 02.8$ \\
$1500 \mathrm{~m}$ & 4 & $03: 58.9 \pm 0: 06.9$ & & 2 & $04: 34.5 \pm 0: 09.2$ \\
$5000 \mathrm{~m}$ & 1 & $14: 58.0 \dagger$ & & 0 & $\mathrm{n} / \mathrm{a}$ \\
\hline †Standard Deviation not reported as $n=1$ & &
\end{tabular}




\section{Types of Interval-training methods used}

Of the 30 respondents, 10 indicated they coached 5-6 running sessions per week, with 1-2 of these most commonly $(n=26)$ prescribed as intervaltraining sessions (Table 4). Twenty-nine of the 30 respondents prescribed the weekly training volume using the total training time $(n=14)$, the total training distance $(n=14)$, or both time and distance depending on the time of the year $(n=1)$. When prescribing IT sessions, long work intervals (> $3 \mathrm{~min}$, $>1500 \mathrm{~m}$ ) and medium work intervals (90 s $-3 \mathrm{~min}$, $500 \mathrm{~m}-1500 \mathrm{~m}$ ) were used by 29 coaches, and short work intervals (<90 s, <500m) were used by 28 coaches.

For middle- (800m - 3000m), long-distance (5000m - half marathon), marathon, ultra-marathon, and fell/trail running events the prescription of IT was reportedly changed by $20,19,11,3$, and 3 coaches, respectively, depending on the time of year. Very few coaches ( $n=1-5$, across all distances) reported they did not change the prescription of IT methods depending on the time of year. A subsection of this question asked coaches to indicate how they altered their prescription of IT for each event distance. Responses were content analysed and resulted in the creation of 9 higher-order themes which are detailed in Table 5, however not all responses fit into these themes. The most common alterations reportedly made by coaches were to increase the intensity and decrease the duration of the work intervals as the season progressed from winter to summer for both middle-distance (800m - 3000m) and long-distance (5000m - half-marathon) events. For the alterations that did not fit into the higherorder themes in middle- and long-distance events, coaches stated that in "winter I use 5k pace", and that alterations are "dependent on the race date". Regarding marathon events, 5 coaches increased the intensity and 4 increased the duration of the work interval as the season progressed from winter to summer (Table 5). Responses that did not fit into the higher-order themes provided by coaches that support athletes in more than one event stated they "change the length, speed, distance, recovery time depending on the training method (I use several) and the periodisation", and alterations "vary depending on whether the season focus is $\mathrm{XC}$, road race league (10k) or half-marathon."

The most common method used to prescribe the intensity of the work interval was race pace $(n=$ 9-10), irrespective of the interval type (Figure 1). The type of interval used dictated the race pace prescribed, with $800 \mathrm{~m}$ race pace most frequently used for short work intervals (SWI) $(n=9), 3000 \mathrm{~m}$ race pace most frequently used for medium work intervals $(\mathrm{MWI})(n=9)$, and $10000 \mathrm{~m}$ race pace most frequently used for long work intervals (LWI) ( $n=11$ ). Respondents using other methods stated they prescribed the work interval intensity based on "a percentage of VAM (maximal aerobic speed)" for SWI, and "speeds that relate to LT4 speeds working below, through and above these speeds" for MWI. For LWI, one coach used " $V O_{2 \max }$ rate", another stated they "base the intensity around and just under athlete's threshold/10mile to half-marathon pace or heart rate based", and one coach stated using "a combination of goal race pace, perceived exertion and recommended paces from Daniels' Running Formula"20.

\section{Running interval-train-} ing sessions per week

\section{n}

week

7

7

10

2

4

$9-10$

0

$\begin{array}{cc}0 & 0 \\ 1-2 & 26 \\ 3-4 & 4 \\ >5 & 0\end{array}$


Table 5. Changes made by coaches to the interval-training prescribed depending on the time of year for each event distance

\section{Interval-training} change

Increase duration

Decrease duration

Increase intensity

Decrease intensity

Increase recovery

Decrease recovery

Increase volume

Decrease volume
Middle distance $(800 \mathrm{~m}$ Long distance $(5000 \mathrm{~m}$ $-3000 \mathrm{~m}$ ) - half marathon)

\section{Marathon}

1
11
11
0
4
0
1
9

1

3
7
9
2
2
1
0

0

4

0

1

9

Ultra-marathon

Fell/Trail running

Respondents provided more than one reason/way they change the interval-training prescription

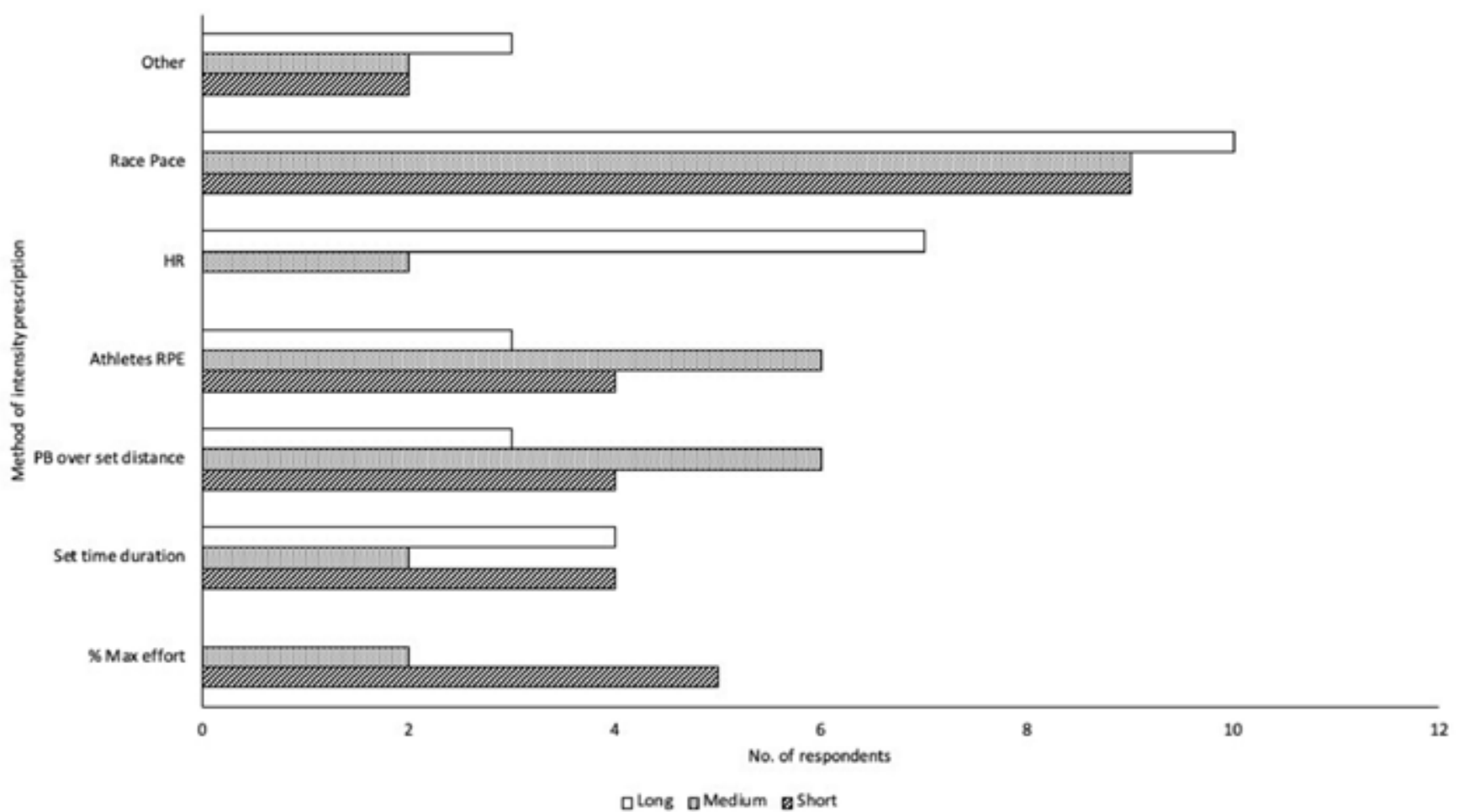

Figure 1. Method of intensity prescription used by coaches. $H R=$ Heart Rate; RPE = Rating of Perceived Exertion; $\%$ Max effort $=$ Percentage of maximum effort; $P B=$ Personal Best. 


\section{Recovery between work intervals}

Active recovery modalities were prescribed most often ( $n=22-26$ ) between work intervals for all interval types. Slow jogs $(n=20)$ were most commonly prescribed between $\mathrm{MWI}$ and LWI, with walks prescribed by 12 coaches between SWI (Table 6). Coaches that indicated prescribing other recovery modalities between SWI stated the modality prescribed "depends on goal of session. Fast jogs if continuous, walk/slow jog if not" along with allowing the athlete to self-select. For LWI, coaches that selected other stated the recovery modality prescribed "Depends on goal of the workout - may be long cruise intervals that aren't difficult, so a fast jog can be sustained during the recovery. Or these may be $\mathrm{VO}_{2 \max }$ reps where a slow jog or walk may be necessary to recover". The intensity of the active recovery modality was prescribed using a set time/ distance $(n=8-9)$ or by allowing the athlete to selfselect the intensity $(n=8)$ most often between MWI and LWI, with the intensity not prescribed $(n=7)$ between SWI. One coach reported they prescribed the intensity of the recovery between SWI "based on full recovery and purpose the intervals sit in the program", and another used "pace" to prescribe the intensity between LWI (Figure 2A). The majority of respondents prescribed recoveries shorter than the work interval between LWI $(n=28)$ and MWI $(n=$ 19). Recovery periods longer than the work interval were prescribed most commonly $(n=15)$ between SWI (Figure 2B).

As part of this question, respondents were asked to provide reasons for the recovery modality prescribed for each type of interval. Reasons provided were content analysed resulting in the creation of 6 higherorder themes which are detailed in Table 7 for short, medium, and long interval types, however, not all these fit into the higher-order themes. The reasons provided for prescribing walks between SWI and MWI that did not fit into the higher-order themes were "to allow the athlete to complete session", "athlete needs more time to recover so walking works well - they can sometimes jog too fast on recovery. Focus of session is doing each individual effort well", and "I prefer them not to clean up the lactic $(\mathrm{H}+)$ between runs to improve tolerance". Reasons for prescribing slow jogs between SWI, MWI and LWI that did not fit into the higher-order themes were "To facilitate the recovery of HR to drop to 125-135bpm before the next rep", "Focus of session is a blend of the effort and the recovery", "Generally have athletes recover at marathon pace or slower so they feel comfortable and associate this pace with lack of effort", and "Continuous runs during off-season, static rest during". A reason for prescribing fast jogs between LWI was for "lactate management via float recovery". Reasons provided for prescribing other recovery modalities between SWI and LWI that did not fit into the higher-order themes included "fast jog if the workout is continuous - perhaps it becomes more of an aerobic stimulus (although the reps likely get slower due to incomplete recovery). Slow jog if high intensity/speed is the goal.", and "depends on goal of the workout - may be long cruise intervals that aren't that difficult, so a fast jog can be sustained during the recovery. Or these may be VO2max reps where a slow jog or walk may be necessary to recover".

\section{Recovery between sets of work intervals}

Between sets of work intervals, active recovery modalities ( $n=19-22)$ of slow jogs $(n=10-16)$ and walks $(n=9)$ were prescribed by respondents most often for all interval types (Table 6). Coaches that indicated prescribing other recovery modalities between sets of work intervals stated the modality is "self-selected by the athlete" or that the recovery is "static initially to refuel then into slow jog" for all interval types. The intensity of the active recovery modality between sets was most commonly prescribed using a set/time distance $(n=7-8)$ or not at all $(n=8-9)$ for all interval types (Figure 2C). The most common recovery time prescribed by coaches between sets was 2-3 min $(n=8-10)$ for all interval types (Figure 2D).

Table 6. Recovery modality prescribed between sets and repetitions of short, medium and long work interval types

\begin{tabular}{|c|c|c|c|c|c|c|}
\hline \multirow{2}{*}{$\begin{array}{l}\text { Recovery modality pre- } \\
\text { scribed }\end{array}$} & \multicolumn{2}{|c|}{ Short Work Intervals } & \multicolumn{2}{|c|}{$\begin{array}{c}\text { Medium Work Inter- } \\
\text { vals }\end{array}$} & \multicolumn{2}{|c|}{ Long Work Intervals } \\
\hline & Sets & Reps & Sets & Reps & Sets & Reps \\
\hline Active - slow jog & 10 & 10 & 15 & 20 & 16 & 20 \\
\hline Active - walks & 9 & 12 & 5 & 4 & 5 & 2 \\
\hline Static & 4 & 1 & 2 & 2 & 1 & 1 \\
\hline Active - fast jog & 0 & 0 & 0 & 1 & 1 & 4 \\
\hline Other & 5 & 4 & 5 & 2 & 5 & 2 \\
\hline
\end{tabular}


A
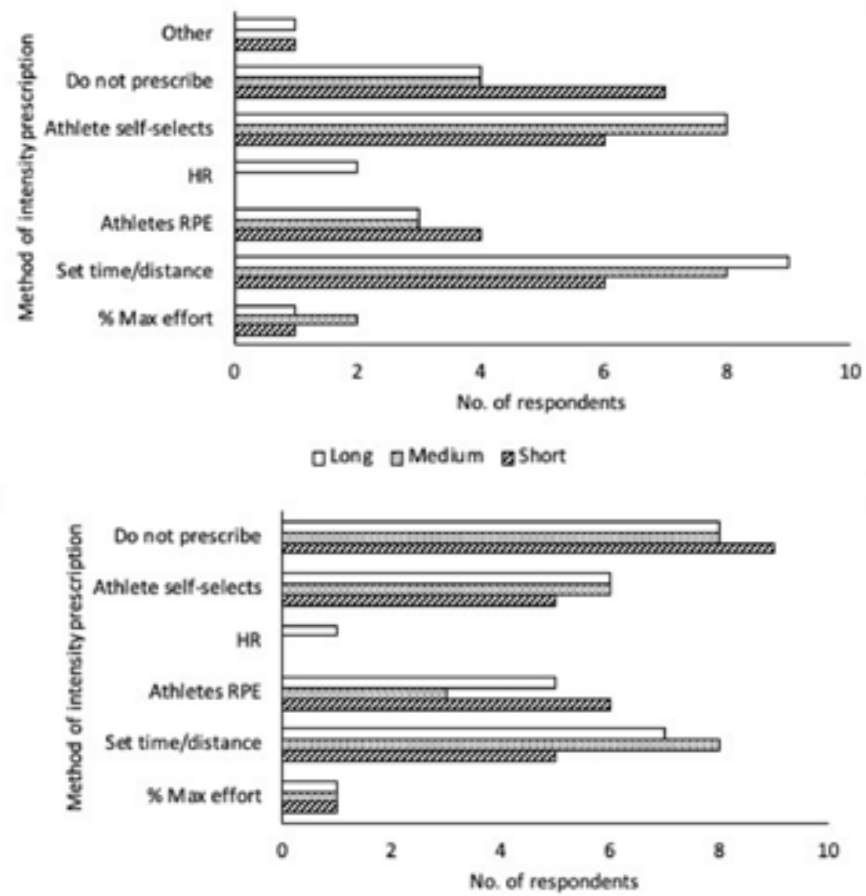

口Long aMedium ashort

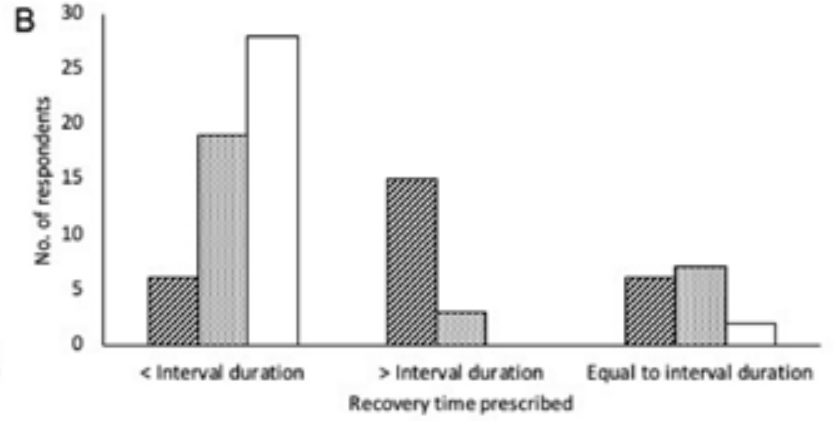

ashort aMedium along

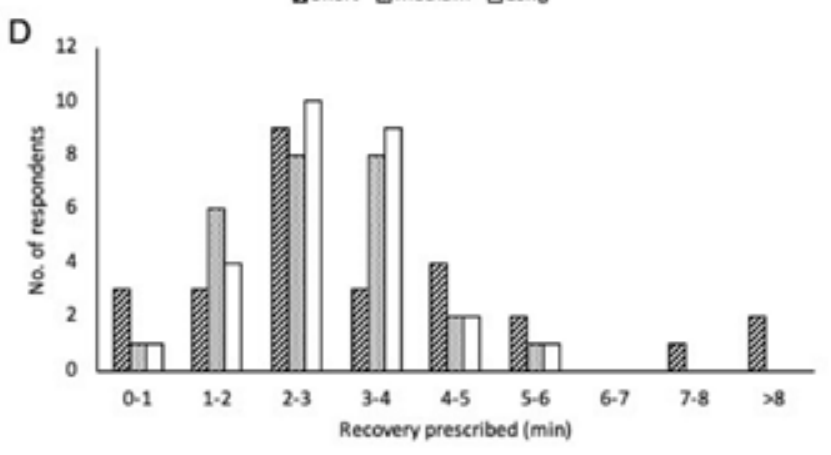

Ghort aMedium olong

Figure 2. Panel A: Intensity prescription of active recovery modalities between work intervals for short, medium and long interval-training types. Panel B: Recovery time prescribed between work intervals for short, medium and long interval-training types. Panel C: Intensity prescription of active recovery modalities between sets of work intervals for short, medium and long interval-training types. Panel D: Recovery time (minutes) prescribed between sets of work intervals for short, medium and long interval-training types. HR = Heart Rate; RPE = Rating of Perceived Exertion; \% Max effort = Percentage of maximum effort; < interval duration = Recovery duration shorter than the work interval; $>$ interval duration $=$ Recovery duration longer than the work interval. 
Table 7. Reasons provided for the recovery modality prescribed between short, medium, and long work intervals.

\begin{tabular}{|c|c|c|c|c|c|c|c|c|c|c|c|c|c|c|c|}
\hline \multirow{2}{*}{$\begin{array}{l}\text { Reason for recovery modality } \\
\text { prescribed }\end{array}$} & \multicolumn{3}{|c|}{ Static } & \multicolumn{3}{|c|}{ Active - walk } & \multicolumn{3}{|c|}{ Active - slow jog } & \multicolumn{3}{|c|}{ Active - fast jog } & \multicolumn{3}{|c|}{ Other } \\
\hline & SWI & MWI & LWI & SWI & MWI & LWI & SWI & MWI & LWI & sWl & MWI & LWI & SWI & MWI & LWI \\
\hline Ensure full recovery & 4 & 2 & 1 & 4 & & 1 & & 4 & 1 & & & & 1 & 1 & \\
\hline Keep athletes moving & & & & 2 & 1 & 1 & 1 & 3 & 2 & & & & & & \\
\hline Facilitate lactic acid removal & & & & 3 & 1 & & & 5 & 2 & & & & & & \\
\hline Maintain aerobic emphasis & 1 & & & & & & & 1 & 4 & & & 1 & & & \\
\hline Ensure appropriate pacing & & & & & 1 & & & 1 & 3 & & 1 & & & & \\
\hline
\end{tabular}

Table 8. Typical structure of short, medium and long interval-training sessions prescribed by coaches

\begin{tabular}{|c|c|c|c|c|c|c|c|c|c|c|}
\hline \multirow[b]{2}{*}{ Interval } & \multirow[b]{2}{*}{ Sets } & \multirow[b]{2}{*}{ Reps } & \multicolumn{3}{|c|}{ Reps } & \multirow[b]{2}{*}{$\begin{array}{l}\text { Rest inten- } \\
\text { sity }\end{array}$} & \multicolumn{2}{|c|}{ Sets } & \multirow{2}{*}{$\begin{array}{c}\sum \text { Work } \\
\text { intervals } \\
(\min )\end{array}$} & \multirow{2}{*}{$\begin{array}{l}\text { Total dura- } \\
\text { tion (min) }\end{array}$} \\
\hline & & & & Work intensity & $\begin{array}{l}\text { Rest dura- } \\
\text { tion (min) }\end{array}$ & & $\begin{array}{l}\text { Rest dura- } \\
\text { tion (min) }\end{array}$ & $\begin{array}{l}\text { Rest inten- } \\
\text { sity }\end{array}$ & & \\
\hline Short & 2 & 5 & $400 m$ & $\begin{array}{c}\text { Max consistent effort (400- } \\
800 \mathrm{~m} \text { pace) }\end{array}$ & $02: 00$ & Walk & 04:00 & Static & $12: 30$ & $32: 30$ \\
\hline Medium & 2 & 5 & $600 \mathrm{~m}$ & $\begin{array}{c}3 \mathrm{~km} \text { pace }(02: 30-03: 00 / \\
\mathrm{km})\end{array}$ & $02: 00$ & Slow Jog & 03:00 & Walk & $16: 30$ & $35: 30$ \\
\hline Long & 1 & 6 & 06:00 min & $\begin{array}{c}\text { 10km pace (03:30-04:00/ } \\
\text { km) }\end{array}$ & 02:00 & Easy jog & - & - & $36: 00$ & 46:00 \\
\hline
\end{tabular}


A

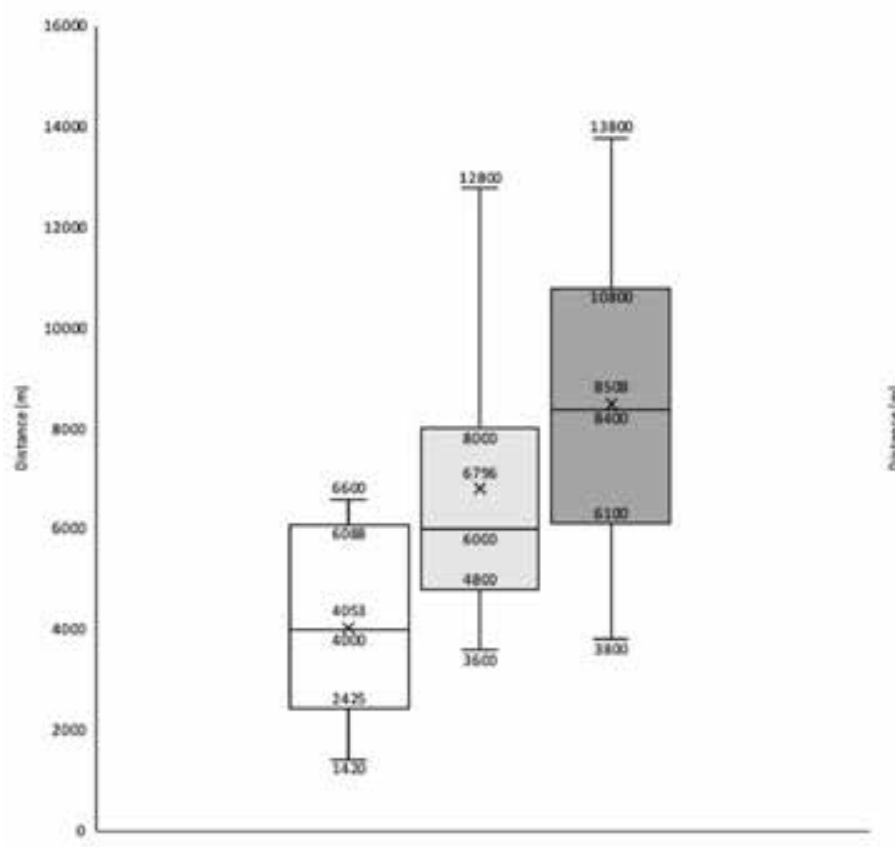

aghere gurtion gions
B

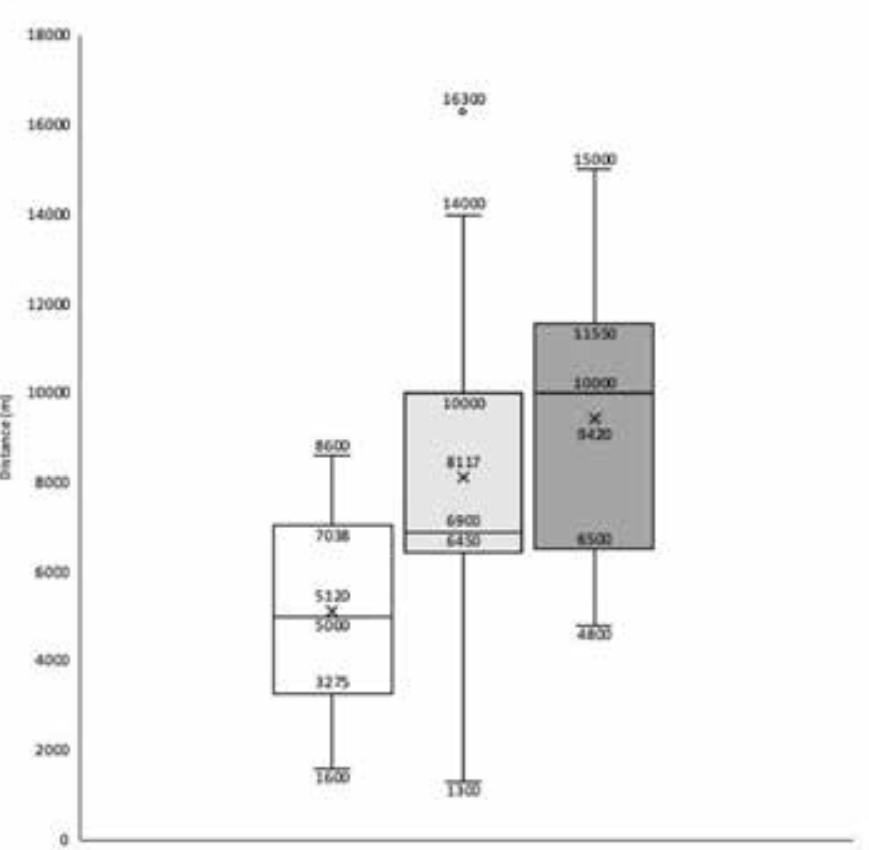

口gant queson quony

Figure 3. Panel A: The volume of running performed during only the work intervals for short, medium and long interval-training sessions. The mean, median, upper and lower quartiles, minimum and maximum distances are displayed for each type of interval-training session. Panel B: The total volume of running performed throughout the entire interval-training session for short, medium and long interval-training sessions. The mean, median, upper and lower quartiles, minimum and maximum distances are displayed for each type of interval-training session.

Table 9. Reasons provided for the type of training method prescribed between consecutive interval-training sessions.

\section{Reason provided}

Facilitate and allow recovery

Increase training volume

Increase base endurance with low intensity

Reduce lower limb loading
Cross training
Circuit

training

\section{LIC run- \\ MII run-}

ning

LII running

$\begin{array}{ll}9 & 2 \\ 6 & 2\end{array}$
ning
2
Strength training
Other

6

1

3

1

3

1

2

1

2 Reasons provided by respondents fit into more than one of the identified higher-order themes.

LIC = Low-intensity continuous; LII = Low-intensity interval; MIC = Moderate-intensity 'threshold' continuous; MII = Moderate-intensity 'threshold' interval. 


\section{Interval-training session structure}

The typical structures of short, medium and long IT sessions are presented in Table 8 . The volume of running performed during only the work intervals was lowest when using SWI and highest when using LWI (Figure 3A). Similarly, the total volume of running performed was greatest during LWI and lowest during SWI (Figure 3B). In all types of IT sessions, $1000 \mathrm{~m}$ of the total volume of running was performed during the rest intervals as active rest.

\section{Time between consecutive Interval-training sessions}

The majority of respondents ( $n=23$ ) reported leaving 2-3 days between consecutive IT sessions. Training sessions were prescribed by 27 coaches between consecutive IT sessions, with only 3 coaches reporting they do not. The reasons provided by the 3 coaches for not prescribing training between consecutive IT sessions were "to allow athlete to recover", "to recover mentally and physically", and "train on weekly club night, alternate interval/ conditioning sessions with a steady run. This is what athletes prefer/want". The type of training prescribed between consecutive IT sessions most often was low-intensity continuous running ( $n=26)$, followed by strength training $(n=15)$ and cross training ( $n$ =14). The one coach reporting "other" stated they prescribed "aquajogging". A subsection of this question asked respondents to detail why they prescribed the reported type of training between consecutive IT sessions. Content analysis resulted in the creation of 5 higher-order themes which are presented in Table 9. Some responses did not fit into these themes, of which, the most common reasons provided for all types of training prescribed between consecutive interval-training sessions were "to achieve other training adaptations", "they are the core of training building, interval is just an element", and "all round fitness and development".

\section{Interval-training session frequency}

Twenty-six coaches reported they would not like to include more IT sessions whereas 4 reported they would. The reasons provided by the 4 coaches indicating they would include more IT sessions into the training regime were; "if I know the correct prescription I can train better my runners", "need to build the athlete's speed endurance", "think it would benefit the group in terms of improving performance, many of the athletes in the group have different goals", and "it depends on the athlete's training age (i.e. number of years of training), their ability to recover, and their goals (finish the race or post a fast time). Based on these factors the number of higher intensity sessions a week will be determined". Responses provided by the 26 coaches indicating they would not include more IT sessions into the training regime were content analysed with the creation of 7 higherorder themes which are presented in Table 10. Some of the responses that did not fit into these broader themes included reasons such as "the athletes I work with are young, intervals are only part of the skill set they need to develop", and "as mainly long distance runners sessions needs to be more focused on distance endurance".

\section{Interval-training methods to improve performance}

The majority of respondents ( $n=19$ ) indicated they believed IT methods improve performance more than other training methods such as continuous training or threshold training, with 9 indicating they believed IT methods improve performance the same as other training methods, and 2 respondents indicated they were unsure. The reasons provided by the 9 coaches who answered "the same" led to two higher-order themes being identified, with 7 answers fitting into the first theme: "necessary to incorporate other training methods", and 3 fitting into the second theme: "need volume at lower intensities". For the coaches who answered "more", 5 higher-order themes were identified which are presented in Table 11. Several responses could not be associated with these higher order themes and included reasons such as "it depends on the capabilities that the athlete needs to develop", "works varied energy systems, drives focus on form and technique", and "the interval workouts are completed in a group setting, there is the social aspect to them and they keep the athletes motivated and focussed for the competitions ahead".

Table 10. Reasons provided by respondents for not wanting to include more interval-training sessions into the training regime.

\begin{tabular}{|c|c|}
\hline Reason provided & $\boldsymbol{n}$ \\
\hline Perform enough already & 11 \\
\hline Risk of injury & 6 \\
\hline Recovery is required & 4 \\
\hline Include other training methods & 3 \\
\hline Risk of overtraining & 3 \\
\hline Increase running volume & 2 \\
\hline
\end{tabular}

Reasons provided by respondents fit into more than one of the identified higher-order themes. 
Table 11. Reasons provided by respondents for why they believe interval-training methods improve performance more than other training methods.

\begin{tabular}{cl} 
Reason provided & $\boldsymbol{n}$ \\
\hline Increases training volume at high intensities & 9 \\
Greater physiological adaptations & 8 \\
Personal experience & 3 \\
Research & 2 \\
\hline
\end{tabular}

Reasons provided by respondents fit into more than one of the identified higher-order

themes.

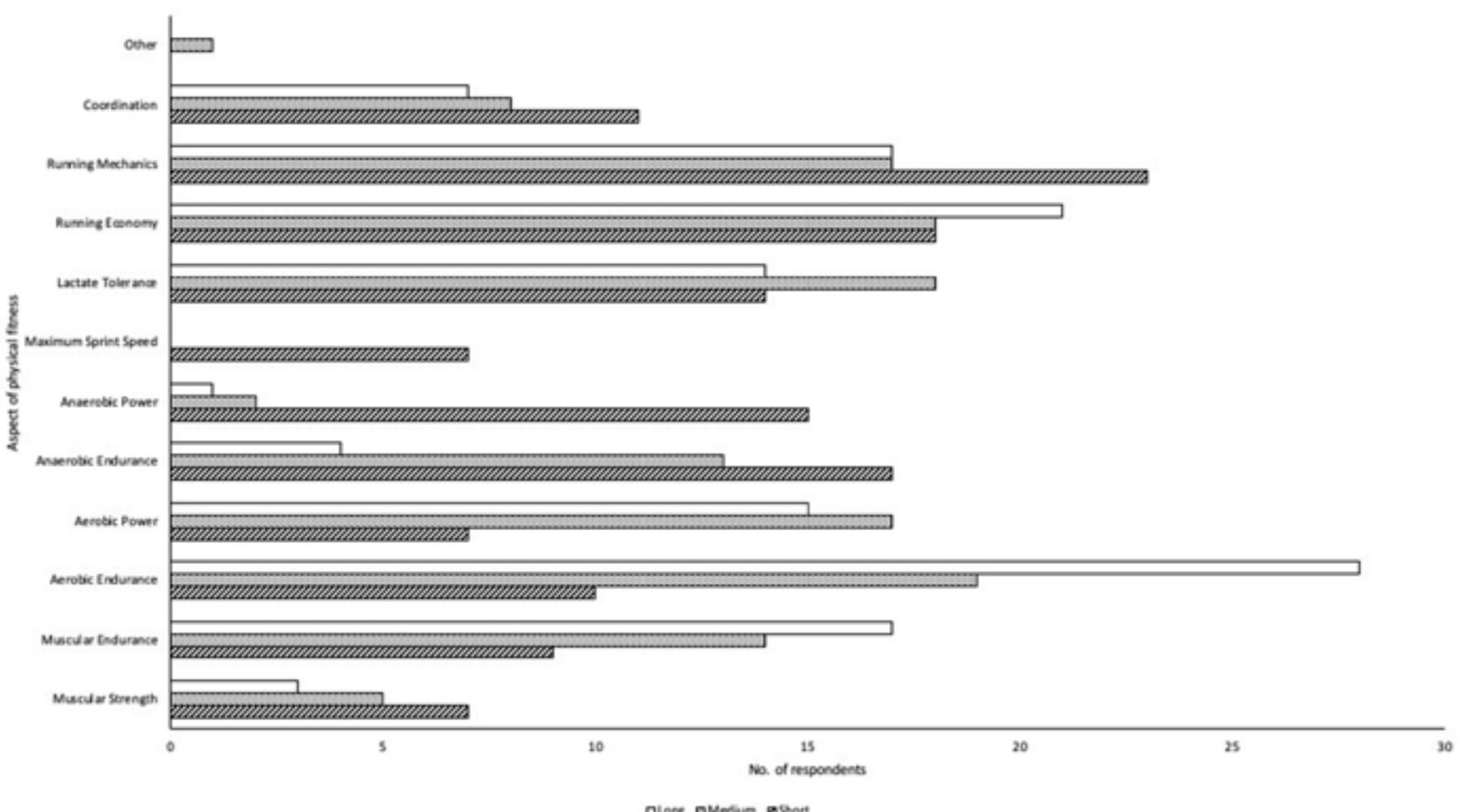

Figure 4. Aspects of physical fitness respondents indicated they are targeting when using short, medium and long work intervals. Respondents indicated targeting more than one aspect of physical fitness for each interval type. 


\section{Aspects of physical fitness}

Coaches were asked to select what aspects of physical fitness they believed they were targeting when using short, medium and long work intervals. The majority of respondents selected they were targeting running mechanics $(n=23)$ when using SWI, whereas the majority of respondents selected they were targeting aerobic endurance $(n=28)$ when using LWI. For MWI, the majority of respondents selected they were targeting aerobic endurance $(n=19)$, lactate tolerance $(n=18)$, running economy $(n=18)$, running mechanics $(n=17)$, and aerobic power $(n=$ 17). One respondent selected "Other" for MWI and stated "I only use this range for athletes that are not used to intervals to get used to the concept, then no more" (Figure 4).

\section{Implementing Interval-training into the program}

The majority of respondents indicated they do not change the prescription of IT depending on the sex of the athlete $(n=22)$, with only 7 indicating they do. Explanations for how respondents altered their IT depending on sex led to 3 higher-order themes being identified. Two explanations fit into the first theme: "accounting for the menstrual cycle", 3 fit into the second theme: "increased running volume for females", and 3 fit into the third theme: "miscellaneous", which were "Reps and sets and recoveries would be different for boys", "Each interval session is different for boys", and "With women I go more off of time running for their intervals instead of distance. For the guys I understand their time running but I'm not going to provide my guys and girls the same exact workout based off of distance with the same recovery because that can have my guys running for 3:00 min and my girls running for 4:15 minutes".

Twenty-one respondents indicated there was nothing unique about the way they prescribed IT, whereas 8 indicated their prescription was unique. Explanations provided by the 8 respondents who indicated their prescription was unique were; "I prefer to use multi-pace and not the same distance within a session", "Grounded in scientific studies and does not follow coaching dogma, not 'unique' but rare", "I would only prescribe interval-training provided the athlete has a base of mileage completed inclusive of slow, steady, threshold pace over a preparation period e.g. 5 weeks at start of prep for cross country", "Less intense for younger athletes", "Some unlimited number of efforts for 10k+ athletes", "The way it is implemented in the macrocycle, each mesocycle (and each athlete) needs a different approach of in- terval work", "Must be based on the athlete's current ability, goals and injury history", and "Making use of power meters with some athletes and power duration curves to have really defined bespoke targets. Learning from other sports (cycling) which is far more bespoke than standard intervals and utilize WKO5 software".

\section{Gaining knowledge of Interval-training methods}

The majority of coaches indicated they learnt about IT methods from their own training $(n=23)$, books $(n=23)$, coaching courses and workshops ( $n=$ 21 ), and scientific journals ( $n=21$ ); with 3 coaches stating they learnt from "physiologists", "graduate school mentor", and "own knowledge/experience with athletes". Respondents ranked scientific journals (39\%), coaching courses and workshops (38\%), and books (32\%), to be their most important sources of education. In contrast, $43 \%$ of respondents ranked friends and club mates as their least important sources of education.

Twenty-four coaches indicated they would like to learn more about IT methods in middle to long-distance running whereas 5 indicated they would not. The 24 respondents provided details relating to what they would like to know leading to five higher-order themes being identified, which are presented in Table 12. Some responses did not fit into these higher-order themes, with coaches stating they would like to learn more about "how to design better developmental progressions for specific distances", "different patterns, posture sessions" and one coach stating "way too many questions to type here. We really know very little. Almost every question still has uncertain answers".

To obtain further information about IT methods, 26 respondents indicated they knew how to obtain this information, however, 4 respondents indicated they did not. The majority of respondents indicated they would obtain further information from coaching courses and workshops $(n=20)$, books $(n=20)$, and scientific journals $(n=19)$, with one coach stating they would "ask a coach of elite athletes if they had the opportunity". 
Table 12. Higher-order themes and representative raw data of what coaches would like to learn about interval-training methods.

\section{Higher-order theme}

Latest research and new ideas

n

Continuous education

6

Periodisation of interval-training

5
ing
Other coaches' use of interval-train-

on the periodization of training over a season and not the use of IT methods as in the present study. The competitive level of athletes coached by respondents most commonly supported in this study were club-, regional- and national-level runners, with the best performance times for various events within $8-28 \%$ and $6-28 \%$ of the 2019 World records for male and female athletes, respectively. The data presented are therefore reflective of the IT practices prescribed by coaches of well-trained middle- to long-distance runners.

\section{DISCUSSION}

The aim of this study was to identify how coaches of well-trained middle- to long-distance runners implement and prescribe interval-training. IT methods were prescribed year-round by the coaches surveyed here, for $1-2$ sessions of the 5-6 training sessions coached per week, irrespective of the event distance. As part of the training regime, coaches utilized a combination of short ( $<90 \mathrm{~s},<500 \mathrm{~m})$, medium (90 s - $3 \mathrm{~min}, 500 \mathrm{~m}-1500 \mathrm{~m}$ ), and long (> 3 min, $>1500 m$ ) work intervals for all event distances. Furthermore, this study is able to report for the first time the typical structure of each type of IT session prescribed by coaches with specific detail of the sets, repetitions, recovery times, intensity, and total volume used.

Over the 9-month period, 30 coaches provided responses to the survey, which is a relatively large sample size for a study examining coaching practices of well-trained middle- to long-distance runners. The use of social networking sites to accrue responses generated over 35,000 impressions, however, the response total of 30 does not reflect such engagement. This perhaps indicates coaches were reluctant to share their practices ${ }^{12}$, were unable to answer the questions within the survey, or did not have the time to complete the survey, although this remains speculative. Previous surveys have reported $20^{21}, 23^{22}$, and $32^{23}$ responses from sport scientists and strength and conditioning (S\&C) coaches supporting elite-level sports teams, however these were related to general S\&C practices rather than specific aspects of training. Similarly, surveys of well-trained endurance runners have reported total responses of $37^{13}, 30^{12}$, and 93 , of which only 16 were classed as elite runners ${ }^{14}$. The runners themselves rather than coaches, provided the responses in the aforementioned studies; therefore, the training performed might not be representative of the training prescribed ${ }^{24}$. A survey of coaches of competitive distance runners (800m to marathon) reported a total of 123 responses ${ }^{24}$, however, this was focused
Irrespective of the event distance athletes were training for, coaches prescribed long, medium, and short work intervals, with $80 \%$ of coaches periodizing the type of work interval throughout the year. Specifically, these coaches prescribed shorter, higher-intensity work intervals as the season progressed from the winter to summer for both middle- and long-distance events. This supports previous findings, reporting coaches prescribed training decreasing in volume and increasing in intensity as the competition date approached $^{24}$. Similarly, more recent studies have reported endurance runners placed a greater emphasis on shorter, higher-intensity work intervals as the competition date approached ${ }^{12,13}$. The inclusion of IT emphasizing high-intensity, short work intervals during a taper has been correlated with greater endurance performance ${ }^{13}$. For middle-distance runners however, the work intensity used was not faster than race pace, whereas for long-distance and marathon runners, work intensities were greater than race pace, which is perhaps a product of the vast differences in race paces of these events. Previously, Kurz et al. (2000) also reported that more IT performed per week during the peaking phase correlated to a better competition performance in Division 1 NCAA cross-country runners. The intensities used during these IT sessions were not reported, however, the total miles run per week and the longest run performed during this peaking phase were lower, along with more rest days taken per week compared with other phases. This perhaps indicates the IT sessions consisted of lower running volumes at higher intensities, therefore requiring more recovery between sessions to be able to adapt and perform the repetitions at the prescribed intensities; due to the greater musculoskeletal and cardiorespiratory demand of performing high-intensity intervals ${ }^{7,25}$.

The use of race-pace was the most commonly reported method to prescribe the intensity of all types of work intervals. The race-pace prescribed however, was dependent upon the type of work interval, with $800 \mathrm{~m}, 3000 \mathrm{~m}$, and $10000 \mathrm{~m}$ race-paces most wide- 
ly used for short, medium, and long work intervals, respectively. Coaches selected 'aerobic endurance' as the physical aspect they were attempting to develop using LWI of 6 min at 10000m race-pace, accumulating a total of 36 min at this pace. The physiological intensity of $10000 \mathrm{~m}$ race-pace has been shown to be approximately $90-95 \%$ of the velocity at $\mathrm{VO} 2 \mathrm{max}\left(\mathrm{VVO}_{2 \max }\right) 3$, falling into the suggested optimal intensity range of $90-100 \% \mathrm{VO}_{2 \max }$ within research to improve $\mathrm{VO}_{2 \max }$ and the maximal steady state (MSS) ${ }^{1,2,7,16}$. In well-trained runners, the lower limit of this range is close to, or slightly above MSS 26, which might not provide the stimulus required to elicit improvements in $\mathrm{VO}_{2 \max }{ }^{2,7}$. Supporting this, $10000 \mathrm{~m}$ race-pace has been reportedly used during threshold, tempo run, and LWI (1000 - 5000m) training sessions in elite-level and world-class long distance runners, with the intensity equating to approximately $82-92 \% \mathrm{HR}_{\max }{ }^{27,28}$. Recent observations have reported that elite marathon runners perform continuous tempo runs and high-volume LWI sessions (e.g. $25-30 \times 400 \mathrm{~m}, 10-15 \times 1000 \mathrm{~m}$ ) at $10000 \mathrm{~m}$ race pace to develop MSS so a high fraction of $\mathrm{VO}_{2 \max }$ can be sustained ${ }^{29}$. Improvements in $\mathrm{VO}_{2 \max }$ using this intensity would rely on the presence of a $\mathrm{VO}_{2}$ slow component which does not occur in well-trained runners, rather a $\mathrm{VO}_{2}$ steady state is achieved 26 . The use of $10000 \mathrm{~m}$ race-pace is therefore suited to improving MSS rather than $\mathrm{VO}_{2 \max }$ due to this intensity being at the lower end of the suggested optimal intensity range for $\mathrm{VO}_{2 \max }$ improvements, with much of the evidence to support this suggestion arising from studies in lesser-trained populations ${ }^{1,2,7}$. The high volume of running performed during LWI sessions is perhaps due to this race pace being synonymous with MSS, allowing coaches to prescribe high running volumes at this intensity without the fatigue associated with IT at high speeds and intensities ${ }^{25,30}$. The structure of the LWI prescribed by coaches appears to be in agreement with research suggestions to effectively develop 'aerobic endurance', with high running volumes able to be performed due to $10000 \mathrm{~m}$ race-pace being relatively sustainable for these runners.

For medium work intervals (MWI) coaches selected 'aerobic endurance' and 'lactate tolerance' as the physical aspects they were attempting to develop. The prescription of $3000 \mathrm{~m}$ race-pace for $\mathrm{MWI}$ has been shown to be approximately $100 \% \vee V^{2} \mathrm{max}^{3,31}$, with the majority of coaches prescribing $400-800 \mathrm{~m}$ ( 90 - $120 \mathrm{~s})$ work intervals at a work: rest ratio of approximately $1: 1$. This prescription might not be sufficient to provide the physiological stimulus necessary to elicit the cardiorespiratory adaptations associated with improvements in $\mathrm{VO}_{2 \max }$ and MSS due to the limited time accumulated at this intensity, especially considering the prescribed work: rest ratio $^{18}$. For the development of 'lactate tolerance', the prescribed work duration is representative of anaerobic 'speed-endurance maintenance' training protocols ${ }^{32}$, however the work intensity and rest prescribed are respectively lower and shorter than that typically used in research. Moreover, coaches prescribed slow jogs and walks between repetitions and sets, respectively, which they stated was to "ensure full recovery" whilst also "maintaining an aerobic emphasis" throughout the session. By contrast, research protocols prescribe static recoveries which can alter the physiological response due to differences in oxygen kinetics when using active recoveries compared to static recoveries ${ }^{33}$. The use of active recoveries maintains a greater $\mathrm{HR}$ and $\mathrm{VO}_{2}$, increasing the contribution from aerobic metabolism that facilitates the removal of blood lactate in comparison to static recoveries ${ }^{34}$. The use of static recoveries during 'speed-endurance maintenance' training perhaps increases the anaerobic contribution, limiting the metabolism and transport of [BLa ] between work intervals providing a greater stimulus for increasing 'lactate tolerance'. Furthermore, 'speed-endurance maintenance' training protocols have been suggested to increase $\mathrm{Na}^{+}, \mathrm{K}^{+}$ATPase pump activity, helping to maintain the membrane potential that would otherwise depolarize due to the loss of $\mathrm{K}^{+}$from the contracting muscle cells contributing to fatigue ${ }^{32}$. Such adaptations contribute to a greater capacity to repeat and maintain high-intensity work bouts, facilitating the development of 'lactate tolerance'32. Such discrepancies between research suggestions and the MWI prescribed by the coaches here could be attributed to coaches attempting to develop multiple physiological aspects at once due to the practical time constraints they find themselves under that are not apparent in research, although this remains speculative.

Short work intervals (SWI) were prescribed at a maximum consistent effort (approximately $<800 \mathrm{~m}$ pace, $\left.115-130 \% \mathrm{VO}_{2 \max }{ }^{31}\right)$ over $400 \mathrm{~m}$, with a work: rest ratio of approximately 1:3. Coaches selected 'running economy', 'running mechanics', and 'anaerobic endurance' as the physical aspects they were attempting to develop with SWI. The SWI prescribed by coaches might not be as effective as those used in research to improve running economy (RE), as supramaximal intervals appear to be effective only during uphill protocols to stimulate neuromuscular adaptations $^{39}$, and cardiopulmonary adaptations effectively stimulated using LWI at intensities $\leq 100 \%$ 
$\mathrm{VVO}_{2 \max }{ }^{35-37}$. These research findings were reported during short-term interventions (6 weeks), whereas the SWI prescribed by coaches are over longer-term interventions ( $>6$ weeks) throughout a season and in combination with other training modalities. Such differences could contribute to the disparity between research recommendations and the SWI prescribed by coaches as acute changes in RE might not have occurred whereas longer-term interventions (> 6 weeks) might have resulted in chronic changes in RE. Similarly, the coaches here prescribe lower work intensities than the near maximal intensity efforts $\left(\geq 130 \% \vee V \mathrm{~V}_{2 \max }\right)$ indicated by research to be effective for developing 'anaerobic endurance'3,32. Such intensities have been shown to tax the capacity of phosphagen and glycolytic energy systems, stimulating the enzymatic adaptations necessary to improve 'anaerobic endurance' 32,38 , along with performance improvements of $3-5 \%$ in $3000 \mathrm{~m}$ and $10000 \mathrm{~m}$ running trials ${ }^{39}$, and $40 \mathrm{~km}$ cycling trials ${ }^{40}$ following 4-9 weeks of speed-endurance training in well-trained runners and cyclists. The maximum consistent intensity prescribed by coaches might not effectively tax the capacity of anaerobic pathways to therefore increase 'anaerobic endurance', despite similar work: rest ratios and work interval durations ( $75 \mathrm{~s}$ ) as those used in research. Rather, the lower intensities paired with rest durations 3-fold the work duration perhaps provides more of an aerobic training stimulus. Coaches stated that the prescribed recovery durations were to ensure a full recovery was achieved between repetitions so that work intervals could be performed at the prescribed intensity every time. By contrast, protocols recommended in research improve 'anaerobic endurance' by gradually accumulating fatigue throughout the session to improve the ability to sustain intense exercise $^{32}$. These high intensity protocols require repeated high velocity running with limited recovery that relies on the recruitment of larger, more fatigable motor-units and muscle fibres to generate the higher forces to accelerate mass ${ }^{41}$, in addition to a greater anaerobic contribution ${ }^{7}$. As a result, the increased metabolic and neuromuscular demand can lead to a loss of running form limiting the volume of high-intensity running able to be sustained within a single session, along with the ability to maintain the high volumes of training typically performed by welltrained runners $3,4,25$ due to the increased recovery time required $\mathrm{d}^{7,30,42}$. The need for increased recovery times when performing interval-training at the high intensities used in research is supported by the abovementioned studies reducing the total training volume by up to $30 \% 39,40$. Maintaining a certain volume of aerobic training was acknowledged as a key part of the training regime, as too great a reduction in volume can negatively affect performance improvements in well-trained endurance athletes ${ }^{39,40,43}$. This perhaps contributes to the lower intensities and longer recoveries of the SWI prescribed by coaches, so that additional training sessions targeting other aspects of physical fitness can be performed alongside IT sessions, without the risk of overtraining ${ }^{35}$.

These 'effective' research recommendations are typically reported following controlled training interventions, where no additional training is performed, or concurrent training is controlled to limit its effects on dependent variables. In addition, research interventions are typically performed in 4-8 week training blocks with 3-4 IT sessions performed per week ${ }^{3,4,16,35}$. In contrast, training sessions prescribed by coaches are targeted at improving various aspects of physical fitness, typically performed concurrently as part of a training meso- and macrocycle for the holistic development of the middleto long-distance runner. The coaches in this study indicated they prescribe 1-2 IT sessions per week, with such sessions performed year-round. The reduced session frequency prescribed in practice is most likely attributable to the recovery-time required between IT sessions so these can be performed at the prescribed intensities; whilst also accounting for the stress imposed from additional training ${ }^{25,35}$. Within scientific literature however, IT protocols prioritize maximizing the time spent at certain physiological intensities and thresholds, with little regard for the stress imposed when performing such sessions, the recovery required to be able to perform at these high intensities, and the stress accumulated from additional training ${ }^{7}$. Furthermore, the logistical and practical constraints placed upon coaches that are not apparent in research limit the time available to develop one particular aspect of physical performance at a time. Rather, coaches are faced with the realities of programming training into the daily lives and routines of their athletes whilst also accounting for competition dates, resulting in training prescriptions that are at a discourse with research suggestions, but perhaps effective in the holistic preparation of the athlete to perform in competition.

To improve performance, most coaches believed IT methods were more effective than other methods such as continuous or threshold training; due to an increased volume of training performed at higher intensities eliciting greater physiological adaptations. These beliefs are supported by research, with greater physiological and performance improvements reported following IT interventions in comparison 
to continuous-training interventions ${ }^{15,17,44}$. Despite the greater improvements from interval-training, the coaches in this survey stated they would not implement more IT into the training regime as enough is performed already and performing more can increase the risk of injury. This statement highlights the need for coaches to appropriately manage the volume of IT performed within the overall training programme so that the risk of injury and overtraining is minimised. Protocols designed and studied in research do not have to consider these practicalities, meaning they might not be appropriate to implement within a holistic training regime as the demands of performing such sessions could adversely affect the ability to perform additional training. Supporting this notion, markers of overtraining were reported when the number of IT sessions was increased from 1 to 3 sessions per week for 4 weeks, despite the total running volume remaining the same throughout ${ }^{38}$.

Interestingly, only 7 of the 29 coaches altered their prescription of IT depending on the sex of the athlete, despite evidence indicating females display an increased fatigue resistance, improved recovery, and higher cardiovascular strain compared to males during IT protocols 45-47. The alterations reportedly made by the coaches in this survey were to either increase the running volume for females or to account for the menstrual cycle. Recent research has indicated that the menstrual cycle can affect cardiorespiratory responses and in turn adaptations to IT 48,49. Such differences between sexes perhaps warrants greater consideration when coaches prescribe IT sessions, although it is acknowledged that a lack of research currently exists for coaches to make evidence-based alterations.

A novel finding of this study is that coaches indicated they learnt about IT methods primarily from their own training and coaching books. When asked to rank the value of their sources of education however, scientific literature, coaching courses and workshops, and books were ranked as the most valuable by $39 \%, 38 \%$, and $32 \%$ of coaches respectively. Despite scientific literature being ranked as the most valuable, the IT sessions prescribed by coaches differ to research suggestions. Moreover, books, coaching courses and workshops are typically informed by research literature further raising the question of why differences between research recommendations and what is prescribed in practice are present. As coaches indicated their own training to be their primary source of education, a combination of this and research recommendations perhaps inform their IT prescription, contributing to the dif- ferences between research and practice, although this remains speculative. Interestingly, coaches indicated they would like to learn more about the latest research and new ideas of prescribing IT methods, with the majority stating they would obtain this information from books, coaching courses and workshops, and scientific journals.

\section{CONCLUSION}

This survey presents a novel insight into the interval-training practices of the coaches of well-trained runners, with the IT sessions typically prescribed consisting of lower intensities, longer recoveries and lower total running volumes than those in research. This is despite coaches indicating scientific journals to be their most valued source of information, highlighting the disconnect between IT protocols that are appropriate to be implemented in practice and those recommended in scientific literature. Another key finding from this survey was that coaches understand and recognise the advantages of IT; however, they prescribe IT sessions that can be completed at the desired volume and intensity without adversely affecting the ability to perform other sessions within the overall training programme. The development of other aspects of physical fitness using other training methods is one of the several practical considerations coaches have to take into account when prescribing IT that are not apparent when designing research studies. Using the responses from the coaches here, research studies can be designed with improved ecological validity to increase the understanding of the effects of IT in well-trained runners. Such studies should be disseminated via books or coaching courses and workshops, in addition to peer-reviewed publications so that novel findings can be accessed by coaches. This could present more opportunities for collaboration between researchers and coaches of well-trained runners for future research, thereby providing ecologically valid conclusions and recommendations that will better inform coaching practice.

\section{ACKNOWLEDGEMENTS}

Not applicable

\section{DECLARATION OF CONFLICTING INTERESTS}

The authors report no conflicts of interest 


\section{FUNDING}

Not applicable

\section{REFERENCES}

1. Midgley AW, McNaughton LR, Jones AM. Training to enhance the physiological determinants of long-distance running performance: Can valid recommendations be given to runners and coaches based on current scientific knowledge? Sport Med 2007; 37: 857-880.

2. Laursen PB, Jenkins DG. The scientific basis for high-intensity interval training: Optimising training programmes and maximising performance in highly trained endurance athletes. Sport Med 2002; 32: 53-73.

3. Billat VL. Interval training for performance: A scientific and empirical practice. Special recommendations for middleand long-distance running. Part I: Aerobic interval training. Sport Med 2001; 31: 13-31.

4. Seiler KS, Kjerland GO. Quantifying training intensity distribution in elite endurance athletes: is there evidence for an 'optimal' distribution? Scand J Med Sci Sport 2006; 16 : 49-56.

5. Brook N, Watts D. Endurance running events. British Athletic Federation, 1992.

6. Lydiard A, Gilmour G. Run, the Lydiard Way: By Arthur Lydiard with Garth Gilmour. Hodder and Stoughton, 1978.

7. Buchheit $M$, Laursen PB. High-intensity interval training, solutions to the programming puzzle: Part 1: Cardiopulmonary emphasis. Sport Med 2013; 43: 313-338.

8. 8. Kenneally M, Casado A, Santos-Concejero J. The effect of periodization and training intensity distribution on middle- and long-distance running performance: A systematic review. Int J Sports Physiol Perform 2018; 13: 11141121.

9. Bale P, Rowell S, Colley E. Anthropometric and training characteristics of female marathon runners as determinants of distance running performance. J Sports Sci 1985; 3: 115-126.

10. Billat VL, Demarle A, Slawinski J, et al. Physical and training characteristics of top-class marathon runners. Med Sci Sport Exerc 2001; 33: 2089-2097.

11. Billat VL, Lepretre P-M, Heugas A-M, et al. Training and bioenergetic characteristics in elite male and female Kenyan runners. Med Sci Sports Exerc 2003; 35: 297-304.

12. Kurz MJ, Berg K, Latin R, et al. The relationship of training methods in NCAA Division I cross-country runners and 10,000-meter performance. J Strength Cond Res 2000; 14: 196-201.

13. Spilsbury KL, Fudge BW, Ingham SA, et al. Tapering strategies in elite British endurance runners. Eur J Sport Sci 2015; 15: 367-373.

14. Karp JR. Training characteristics of qualifiers for the U.S. Olympic marathon trials. Int J Sports Physiol Perform 2007; 2: 72-92.

15. Bacon AP, Carter RE, Ogle EA, et al. VO2max trainability and high intensity interval training in humans: a meta-analysis. PLoS One 2013; 8: e73182.

16. Midgley AW, McNaughton LR, Wilkinson M. Is there an optimal training intensity for enhancing the maximal oxygen uptake of distance runners? Empirical research findings, current opinions, physiological rationale and practical recommendations. Sport Med 2006; 36: 117-132.

17. Milanović Z, Sporiš G, Weston M. Effectiveness of high-intensity interval training $(\mathrm{HIT})$ and continuous endurance training for VO2max improvements: A systematic review and meta-analysis of controlled trials. Sport Med 2015; 45 : 1469-1481.

18. Parmar A, Jones TW, Hayes PR. The dose-response relationship between interval-training and $\mathrm{VO} 2 \mathrm{max}$ in well-trained endurance runners: A systematic review. J Sports Sci. Epub ahead of print 2021. DOI: 10.1080/02640414.2021.1876313.

19. Patton MQ. Qualitative evaluation and research methods, 2nd ed. Thousand Oaks, CA, US: Sage Publications, Inc, 1990

20. Daniels J. Daniels' Running Formula. Human Kinetics, Incorporated, 2013.

21. Simenz CJ, Dugan CA, Ebben WP. Strength and conditioning practices of National Basketball Association strength and conditioning coaches. J Strength Cond Res 2005; 19: 495-504.

22. Ebben WP, Carrol RM, Simenz CJ. Strength and conditioning practices of national hockey league strength and conditioning coaches. J Strength Cond Res 2004; 18: 889-897.

23. Gee TI, Olsen PD, Berger NJ, et al. Strength and Conditioning practices in rowing. J Strength Cond Res 2011; 25: 668-682.

24. Hewson DJ, Hopkins WG. Prescribed and self-reported seasonal training of distance runners. J Sports Sci 1995; 13: 463-470.

25. Stöggl TL, Sperlich B. Editorial: Training intensity, volume and recovery distribution among elite and recreational endurance athletes. Front Physiol 2019; 10: 1-4.

26. Billat $V$, Binsse V, Petit $B$, et al. High level runners are able to maintain a VO2 steady-state below VO2max in an all-out run over their critical velocity. Arch Physiol Biochem 1998; 106: 38-45.

27. Casado A, Hanley B, Santos-Concejero J, et al. World-class long-distance running performances are best predicted by volume of easy runs and deliberate practice of short-interval and tempo runs. J Strength Cond Res 2019; 1.

28. Tjelta LI. Three Norwegian brothers all European $1500 \mathrm{~m}$ champions: What is the secret? Int J Sports Sci Coach 2019; 14: 694-700.

29. Jones AM, Kirby BS, Clark IE, et al. Physiological demands of running at 2-hour marathon race pace. J Appl Physiol 2021; 130: 369-379.

30. Garciá-Pinillos F, Párraga-Montilla JA, Soto-Hermoso VM, et al. Acute metabolic, physiological and neuromuscular responses to two high-intensity intermittent training protocols in endurance runners. Isokinet Exerc Sci 2016; 24: 99-106.

31. Sandford GN, Stellingwerff T. "Question Your Categories": the misunderstood complexity of middle-distance running profiles with implications for research methods and application. Front Sport Act Living 2019; 1: 28.

32. laia FM, Bangsbo J. Speed endurance training is a powerful stimulus for physiological adaptations and performance improvements of athletes. Scand J Med Sci Sports 2010; 20: 11-23.

33. Abderrahman BA, Zouhal $\mathrm{H}$, Chamari $\mathrm{K}$, et al. Effects of recovery mode (active vs. passive) on performance during a short high-intensity interval training program: a longitudinal study. Eur J Appl Physiol 2013; 113: 1373-1383.

34. Dorado C, Sanchis-Moysi J, Calbet JAL. Effects of recovery mode on performance, $\mathrm{O} 2$ uptake, and $\mathrm{O} 2$ deficit during high-intensity intermittent exercise. Can J Appl Physiol 2004; 29: 227-244.

35. Billat VL, Flechet $\mathrm{B}$, Petit $\mathrm{B}$, et al. Interval training at VO2max: effects on aerobic performance and overtraining markers. Med Sci Sports Exerc 1999; 31: 156-163.

36. Barnes KR, Hopkins WG, McGuigan MR, et al. Effects of different uphill interval-training programs on running economy and performance. Int J Sports Physiol Perform 2013; 


\section{8: 639-647.}

37. Franch J, Madsen K, Djurhuus MS, et al. Improved running economy following intensified training correlates with reduced ventilatory demands. Med Sci Sport Exerc 1998; 30: 1250-1256.

38. Iaia FM, Hellsten $Y$, Nielsen JJ, et al. Four weeks of speed endurance training reduces energy expenditure during exercise and maintains muscle oxidative capacity despite a reduction in training volume. J Appl Physiol 2009; 106: 73-80.

39. Bangsbo J, Gunnarsson TP, Wendell J, et al. Reduced volume and increased training intensity elevate muscle $\mathrm{Na}+-\mathrm{K}+$ pump a2-subunit expression as well as short- and long-term work capacity in humans. J Appl Physiol 2009; 107: 1771-1780.

40. 40. Laursen PB, Shing CM, Peake JM, et al. Interval training program optimization in highly trained endurance cyclists. Med Sci Sport Exerc 2002; 34: 1801-1807.

41. Edgett BA, Foster WS, Hankinson PB, et al. Dissociation of increases in PGC-1 $a$ and its regulators from exercise intensity and muscle activation following acute exercise. PLoS One 2013; 8: e71623.

42. Buchheit $M$, Laursen PB. High-intensity interval training, solutions to the programming puzzle: Part II: Anaerobic energy, neuromuscular load and practical applications. Sport Med 2013; 43: 927-954.

43. Iaia FM, Thomassen M, Kolding $\mathrm{H}$, et al. Reduced volume but increased training intensity elevates muscle $\mathrm{Na}+\mathrm{K}+$ pump a1-subunit and NHE1 expression as well as shortterm work capacity in humans. Am J Physiol Integr Comp Physiol 2008; 294: R966-R974.

44. Stoggl T, Sperlich B. Polarized training has greater impact on key endurance variables than threshold, high intensity, or high volume training. Front Physiol 2014; 5: 33.

45. Laurent CM, Green JM, Bishop PA, et al. Effect of gender on fatigue and recovery following maximal intensity repeated sprint performance. J Sports Med Phys Fitness 2010; 50: 243-253.

46. Laurent CM, Vervaecke LS, Kutz MR, et al. Sex-specific responses to self-paced, high-intensity interval training with variable recovery periods. J Strength Cond Res 2014; 28 : 920-927.

47. Schmitz B, Niehues H, Thorwesten L, et al. Sex differences in high-intensity interval training-Are HIIT protocols interchangeable between females and males? Front Physiol 2020; 11: 38.

48. Rael B, Alfaro-Magallanes VM, Romero-Parra N, et al. Menstrual cycle phases influence on cardiorespiratory response to exercise in endurance-trained females. Int J Environ Res Public Health 2021; 18: 860.

49. McNulty KL, Elliott-Sale KJ, Dolan E, et al. The effects of menstrual cycle phase on exercise performance in eumenorrheic women: A systematic review and meta-analysis. Sport Med 2020; 50: 1813-1827. 